Acta Crystallographica Section A

Foundations of Crystallography

ISSN 0108-7673

Received 20 October 2006

Accepted 3 May 2007

C 2007 International Union of Crystallography Printed in Singapore - all rights reserved

\section{Diffractive imaging for periodic samples: retrieving one-dimensional concentration profiles across microfluidic channels}

\author{
Oliver Bunk, ${ }^{\mathrm{a} *}$ Ana Diaz, ${ }^{\mathrm{a}} \ddagger$ Franz Pfeiffer, ${ }^{\mathrm{a}}$ Christian David, ${ }^{\mathrm{a}}$ Bernd Schmitt, ${ }^{\mathrm{a}}$ \\ Dillip K. Satapathy ${ }^{a}$ and J. Friso van der Veen ${ }^{a, b}$
}

\begin{abstract}
${ }^{\text {a } R e s e a r c h ~ D e p a r t m e n t, ~ S y n c h r o t r o n ~ R a d i a t i o n ~ a n d ~ N a n o t e c h n o l o g y, ~ P a u l ~ S c h e r r e r ~ I n s t i t u t, ~} 5232$ Villigen PSI, Switzerland, and ${ }^{\mathbf{b}}$ ETH Zurich, 8093 Zürich, Switzerland. Correspondence e-mail: oliver.bunk@psi.ch
\end{abstract}

\begin{abstract}
A technique has been developed that allows determination of the concentration profiles of colloidal solutions or any kind of fluid under confinement. Currently, submicrometre-wide channels are sampled with a resolution in the $10 \mathrm{~nm}$ range. The method comprises regular arrays of microfluidic channels and onedimensional X-ray phase-retrieval techniques for the analysis of small-angle $\mathrm{X}$-ray diffraction from the array structures. Recording the X-ray diffraction data requires a low dose on each individual channel since the sum of the signals from all channels is detected. The determined concentration profiles represent the ensemble average rather than individual entities and are obtained in a modelindependent way. As an example, amplitude and phase of the exit field and concentration profiles for a colloidal fluid within confining channels of different widths are shown.
\end{abstract}

\section{Introduction}

Geometrical confinement can dramatically alter bulk properties of fluids like viscosity and density. This has important implications for fundamental processes such as lubrication, friction, crystallization and transport through narrow pores.

Whereas oscillatory surface forces have been measured for many confined liquids (Israelachvili, 1991, 1992), structural information is scarce since the confinement geometry makes the fluid inaccessible to most structural probes. Optical videomicroscopy requires the system to be transparent and to have sufficiently large and slow particles. Transmission electron microscopy is restricted to fluids with very low vapor pressure, while for atomic force microscopy the generally unknown shape of the cantilever tip restricts applications in this field. Determining with high resolution the density profile of a fluid across the confining container has so far remained impossible, despite some promising proof-of-principle results using X-ray wave guiding (Zwanenburg et al., 1999) and X-ray reflectivity (Seeck et al., 2002).

Instead of investigating single fluid containers, our approach is to use an array of microfluidic channels etched into a silicon chip acting as a diffracting grating structure in small-angle X-ray scattering (SAXS) experiments. This approach has several advantages: by studying many identical containers at the same time, the ensemble average rather than a single entity is investigated, and the scattered X-ray signal is

‡ Present address: European Synchrotron Radiation Facility, BP220, 38043 Grenoble CEDEX, France much stronger. The set-up is insensitive to perturbations such as vibrations, since the dimensions of the fluid containers are not variable. Different dimensions of the confining channels are simply chosen by having a set of different arrays on the same sample, i.e. the same chip. The $\mathrm{X}$-ray dose per sample volume is significantly reduced in comparison to studies of single containers.

The SAXS diffraction patterns of the gratings were analyzed in a model-independent manner. Fourier transformation of the recorded intensities of the grating orders cannot yield density profiles because of the well known phase problem, i.e. the loss of the X-ray phase information in the recorded data. To retrieve the phase information, iterative algorithms turned out to be very useful. However, it has been shown that phase retrieval in one-dimensional problems generally is non-unique, while in two-dimensional problems multiple solutions are less abundant (Bruck \& Sodin, 1979). Nevertheless, there are certain cases reported in which a onedimensional phase-retrieval is possible (Walther, 1963; Vartanyants et al., 2000; Elser, 2003).

For two- and three-dimensional lensless imaging, iterative phase retrieval has proven to be very helpful. Starting from first proof-of-principle studies (Miao et al., 1999) and the imaging of dried Escherichia coli bacteria (Miao et al., 2003), followed by freeze-dried yeast cells (Shapiro et al., 2005; Thibault et al., 2006), lead nanocrystals (Pfeifer et al., 2006) and the reconstruction of the complex field at the focus of a Fresnel zone plate (Quiney et al., 2006), this leads towards three-dimensional imaging of a pyramid-like test object 
(Chapman et al., 2006) and many more impressive results than can be cited here. There is a fundamental difference between these lensless imaging studies and the technique reported here, apart from the dimensionality: the earlier investigations were based on fully coherent illumination of the sample, i.e. used a minor part of the incident flux. The present study uses an only partially coherent macroscopic beam $(0.5 \times 0.5 \mathrm{~mm})$ and thereby most of the incident flux. In this sense, the current phase-retrieval problem is closer to the crystallographic version with only partially coherent illumination, albeit that the atomicity constraint used in crystallography does not apply in the present case.

This article is organized as follows. We start by describing the experimental set-up for the new microfluidic array phaseprofiling (MAPP) technique and the initial treatment of the recorded data. We then present the iterative phase-retrieval algorithm that allows determination of amplitude and phase of the exit field and thereby concentration profiles across the microfluidic channels in a model-independent way. Finally, examples with experimental data are presented to demonstrate the applicability of the technique. For a model-dependent kinematic scattering approach to determine the concentration profile, we refer to Diaz (2006) and Diaz \& van der Veen (2007).

\section{Experimental set-up}

The experimental set-up is schematically shown in Fig. 1. The key component is a silicon $(\mathrm{Si})$ chip with microfluidic arrays, i.e. periodic arrangements of channels with rectangular cross section and of identical width and height. The high-aspectratio microfluidic arrays were fabricated by anisotropic wet etching of $\langle 110\rangle$-oriented silicon substrates in a potassium hydroxide solution. The process involved a double-layer mask

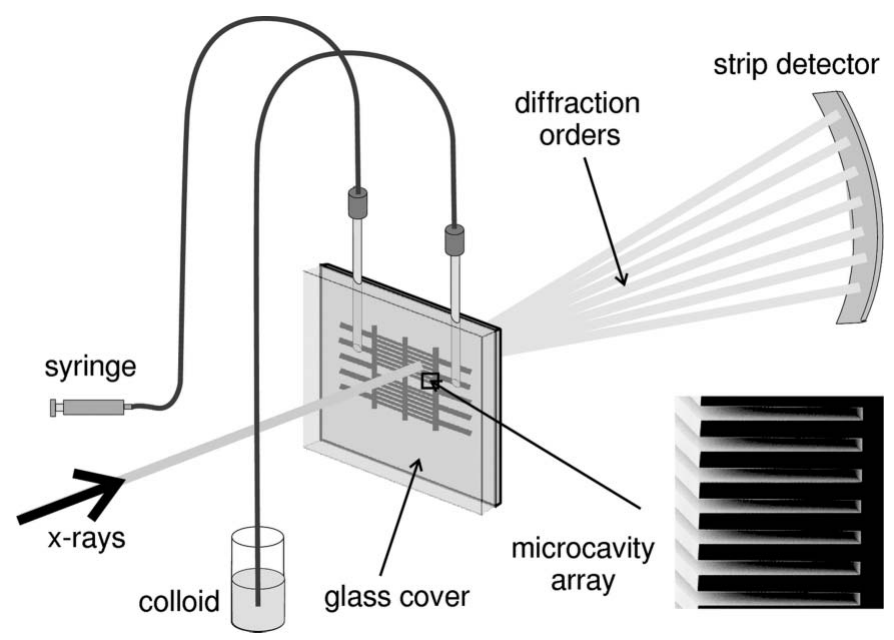

Figure 1

Experimental set-up. The X-ray beam impinges under normal incidence onto the sample chip on which several different microfluidic arrays were prepared. The sample chip is covered with a glass plate to avoid evaporation. The diffracted intensity is recorded with a high-resolution microstrip detector. The inset shows a SEM image of a microfluidic array. created by thermal oxidation, chromium deposition and electron-beam lithography. Using several etching steps, microfluidic arrays of high perfection were fabricated. Details are described elsewhere (Diaz et al., 2005).

The arrays typically have a period on the order of $1 \mu \mathrm{m}$, a channel width of several hundred nanometres, and a height of 5-10 $\mu \mathrm{m}$. For example, the data described in $\$ 5$ were recorded for a chip with microfluidic arrays of $0.50,0.75,1.00$ and $1.25 \mu \mathrm{m}$ period and channel widths in the range of 280 to $615 \mathrm{~nm}$. The height of the structures was approximately $6 \mu \mathrm{m}$. A scanning-electron-microscopy (SEM) image of such a microfluidic array is shown in the inset of Fig. 1.

The channel width of these arrays is not tunable. To overcome this limitation, several microfluidic arrays with different widths of the constituent channels are etched on the same chip. By simply translating the silicon chip it can be chosen which array structure and thereby which channel width is illuminated by the synchrotron beam.

The microfluidic arrays are placed under normal incidence in the monochromatic X-ray beam. The data discussed in this article were recorded at an X-ray energy of $11.804 \mathrm{keV}$, corresponding to a wavelength of $1.050 \AA$, at the Materials Science beamline of the Swiss Light Source, Paul Scherrer Institut (Patterson et al., 2005). The arrays act as diffraction gratings, and the intensities of the diffraction orders for each grating were recorded with a high-resolution one-dimensional microstrip detector (Schmitt et al., 2004). It is a direct detection photon counting system with silicon strips of $50 \mu \mathrm{m} \times 8 \mathrm{~mm}$ area and $300 \mu \mathrm{m}$ thickness. We used three modules with 1280 strips each. The detector-to-sample distance was $5 \mathrm{~m}$ and the X-ray beam was focused onto the detector. The resulting angular resolution was $10 \mu \mathrm{rad}$ $\left(6 \times 10^{-4} \mathrm{~nm}^{-1}\right)$.

Combining three diffraction patterns obtained with different attenuation settings and with or without beam-stop for the central part of the diffraction pattern allowed us also to measure the low-order diffraction intensities, including the zeroth order, which eliminates the need for special treatment of missing low-frequency data (Shapiro et al., 2005; Thibault et al., 2006). The total exposure time for each microfluidic array was $135 \mathrm{~s}$. This time already includes a triple repeat of each exposure used to filter out spurious counting events by taking the median value of the recorded intensities.

\section{Initial data treatment}

The inaccessible regions between detector modules and spurious counting events were corrected by a symmetrization and averaging process that uses the mirror symmetry of the grating diffraction from the microfluidic arrays. A periodic array of Gaussian peaks with the same width but individual intensities on an interpolated background was fitted to the diffraction pattern for each grating in order to retrieve the integrated intensities of the array diffraction peaks with high precision. These integrated intensities are the input values for the following data analysis. 
An example is shown in Fig. 2. The intensities are plotted as a function of the momentum transfer $q$. The detector pixel numbers $n_{\text {det }}$, with $n_{\text {det }}=0$ at the direct-beam position, are converted to momentum transfer using Bragg's law with wavelength $\lambda=1.050 \AA$, absolute value of the wave vector $k=2 \pi / \lambda$, detector pixel size $s_{\text {det }}=50 \mu \mathrm{m}$ and sample-to-detector distance $d_{\mathrm{det}}=5.03 \mathrm{~m}: q=2 k \sin \left[(1 / 2) \arctan \left(n_{\mathrm{det}} s_{\mathrm{det}} / d_{\mathrm{det}}\right)\right] \approx$ $k n_{\text {det }} s_{\text {det }} / d_{\text {det }}$.

The broad background is attributed to diffuse scattering as will be described in more detail in a separate publication concerning two-dimensional X-ray scattering data for these systems.

\section{Data analysis}

Propagation of X-rays through the microfluidic array changes both phase and amplitude of the incident field. This is described by the real part $\delta$ and the imaginary part $\beta$ of the refractive index $n$ :

$$
n=1-\delta+i \beta \text {. }
$$

The goal is to retrieve the complex-valued field at the exit of the grating in a model-independent unique way from the measured amplitudes. Using the known $\delta$ values, we can calculate the density profile of the fluid across the microfluidic channels.

We are using a coordinate system in which $x$ is across the microfluidic channels, $y$ along the channels and $z$ along the propagation direction of the X-rays towards the detector. The diffraction measurements average over $y$. The origin $x=z=0$ is in the center of a channel at the exit of the grating.

\subsection{Phase retrieval}

The intensities $I$ measured in the far-field Fraunhofer regime are proportional to the squared absolute value of the Fourier transformation $\mathcal{F}$ of the complex-valued field $E(x, z=0)$ at the exit of the grating structure:

$$
I=|\mathcal{F}[E]|^{2} .
$$

We are dealing with discrete data of $N$ intensities and therefore the Fourier transform $\mathcal{F}$ will practically be implemented as a discrete fast Fourier transformation.

The goal of the data analysis is retrieving the field from the measured intensities even though the phase information is lost in the measurement process.

4.1.1. Resolution. The field $E(x)$ is sampled in steps of $p / N$, where $p$ is the grating period. The reciprocal-space frequencies $f$ are consequently sampled in steps of $f=1 / p$ up to the maximum frequency of $f_{\max }=N / p$.

The theoretical resolution of the method is inversely proportional to the number of grating diffraction peaks recorded since the pixel size of the reconstructed field is $p / N$. As many similar solutions are averaged, high-frequency noise will average out and one can estimate the true solution from the power spectrum of the average solution.
Please note that in a typical plot like the one in Fig. 2 only $(N-1) / 2+1$ peaks are shown due to the mirror symmetry of the data about the zeroth order.

4.1.2. Solution set. In the near-field Fresnel regime, the field at a distance $z$ from the grating exit is well approximated by multiplying with the Fresnel propagator $P_{z}$ in Fourier space,

$$
E(x, z)=\mathcal{F}^{-1}\left\{P_{z}(f) \mathcal{F}[E(x, 0)]\right\},
$$

where the Fresnel propagator without constant pre-factors is defined as

$$
P_{z}=\exp \left(-i \pi \lambda z f^{2}\right)=\exp \left[-i z q_{x}^{2} /(2 k)\right]
$$

A multiplication in Fourier space is usually computationally easier to implement than a convolution in direct space.

One has to be aware of the fact that all fields $E(x, z)$ at different positions $z$ along the propagation direction, see Fig. 3 for examples, will result in the same far-field diffraction pattern since the Fourier transformation of these fields differs only in phase rather than amplitude - the phase factor in reciprocal space being the Fresnel propagator $P_{z}$. This means that an unconstrained phase-retrieval algorithm finds an infinite number of complex-valued solutions which all are consistent with the measured intensities. The possible solutions include back-propagation results for negative $z$ in equation (3). The back-propagation fields are unphysical in the sense that they do not physically exist. Nevertheless, it is important to consider these solutions. Only the constraints imposed on an iterative phase-retrieval algorithm force convergence in a specific plane, the focal plane of the reconstruction. For an extended object of length $d$, the Fresnel fringes are present at all positions but are minimized around
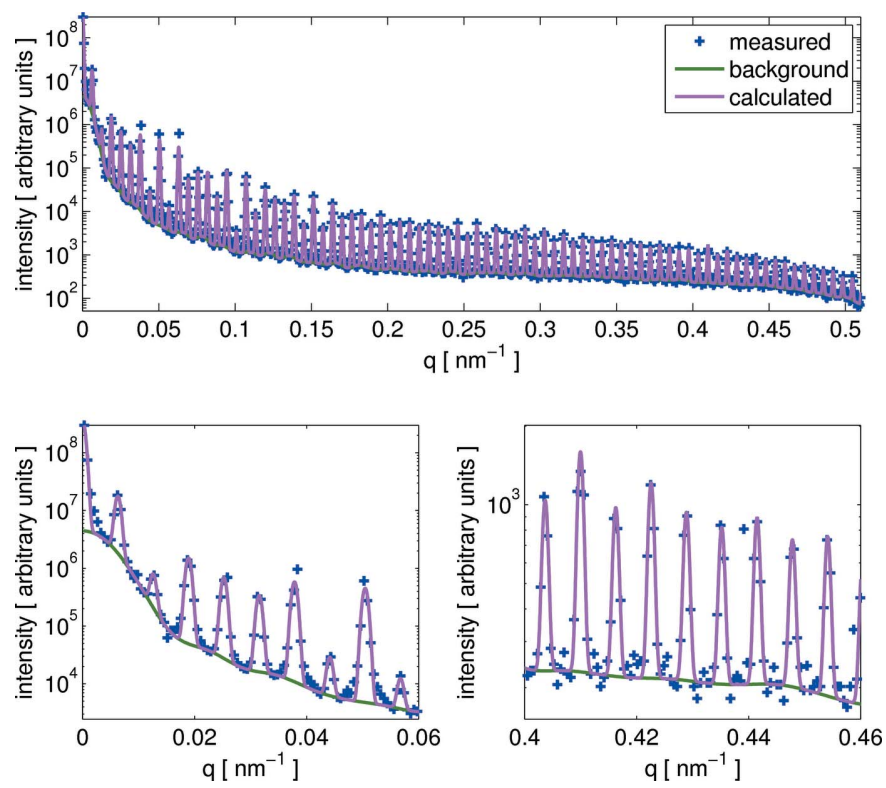

Figure 2

Peak integration. For a grating with $1 \mu \mathrm{m}$ period, the complete diffraction pattern (top) and two subregions (bottom) are shown together with a periodic array of Gaussian peaks on an interpolated background that has been fitted to the diffraction data. The intensity axes are scaled logarithmically. 
the center of the object at $z \approx-d / 2$ (cf. Fig. 3), i.e. the field at the end of the object propagated back through free space by half the length of the object is obtained (Spence et al., 2002; Thibault et al., 2006; Chapman et al., 2006). Phase retrieval with tight constraints will yield the halfway back-propagated field since the constraints will minimize the Fresnel fringes. This is the best approximation of the exit field within the thinobject approximation, even though it has no physical counterpart.

4.1.3. Algorithms. To retrieve the phase of a wavefield from the absolute values of its Fourier transform, iterative phaseretrieval algorithms have proved to be beneficial. These algorithms typically iterate with Fourier transformations between direct and reciprocal space and apply in each domain constraints derived from knowledge about the system. In Fourier space, this simply means that the amplitudes are known from the measured intensities, see equation (2). In direct space, this can for example mean that parts of the object or its range of possible values are known.

For the present case of retrieving one-dimensional density profiles from diffraction data, we attempted a combination of standard iterative phase-retrieval algorithms, namely Gerchberg-Saxton's error-reduction algorithm (Gerchberg \& Saxton, 1972), this algorithm with the phase-selection option (Gerchberg, 1986) and Fienup's hybrid input-output algorithm (Fienup, 1982). It turns out that the convergence towards a unique solution is performed by the iterations using the Gerchberg-Saxton error-reduction algorithm and that using this algorithm alone is sufficient. Nevertheless, the hybrid input-output algorithm was included to ensure that no stagnation occurs, even though in the present case it does not converge towards a stable solution. The phase-selection option was used to damp phase changes and thereby to avoid oscillatory behavior. This was done by using the average of the phases before and after an iteration rather than only the phase after each iteration. But the effect of this modification on the convergence of the algorithm appears to be minute.

For the examples presented in this article, we applied the following scheme. We start with a random solution and 15 iterations of the hybrid input-output algorithm, followed by 20 iterations of the Gerchberg-Saxton algorithm with the phase-selection option repeated five times. We then apply three cycles of 15 iterations of the hybrid input-output algorithm, followed by 50 iterations of the Gerchberg-Saxton algorithm. These are in total 370 iterations with the different algorithms. The method is computationally fast since we are dealing with a one-dimensional data set.

To monitor the convergence of the algorithm, we calculate the mean-squared deviation $\chi^{2}$ of the $N$ amplitudes $\sqrt{I_{n}}$ in Fourier space [equation (2)] from the measured ones for each iteration $i$ :

$$
\chi^{2} \equiv \frac{1}{N} \sum_{n=1}^{N}\left(\sqrt{I_{n}}-\sqrt{I_{n, \text { meas }}}\right)^{2}
$$

The full number of iterations is always performed according to the above scheme and finally the solution with the smallest $\chi^{2}$ is selected. This solution is virtually identical to the result of the last iteration and therefore the monitoring of $\chi^{2}$ has only one practical application, namely finding the right object constraint region as described in §4.1.5.

4.1.4. Parameter spaces. It is sometimes assumed that the mirror symmetry of the grating diffraction pattern leads to 'trivial' phases, i.e. multiples of $\pi$ in reciprocal space, thereby significantly simplifying the problem. In the present case, this assumption is not valid due to the complex-valued refractive index, i.e. absorption cannot be neglected ( $c f$. also Fig. 8).

The periodic translation-invariant part of the array structure, i.e. a single channel, is mirror symmetric. If we start with a symmetric field $E(x)=E(-x)$ after a single channel, then its Fourier transform, given by

$$
\begin{aligned}
\mathcal{F}[E(x)] & =\int_{-\infty}^{\infty} E(x) \exp (-i q x) \mathrm{d} x \\
& =\int_{0}^{\infty} E(-x) \exp (+i q x) \mathrm{d} x+\int_{0}^{\infty} E(x) \exp (-i q x) \mathrm{d} x \\
& =2 \int_{0}^{\infty} E(x) \cos (q x) \mathrm{d} x,
\end{aligned}
$$
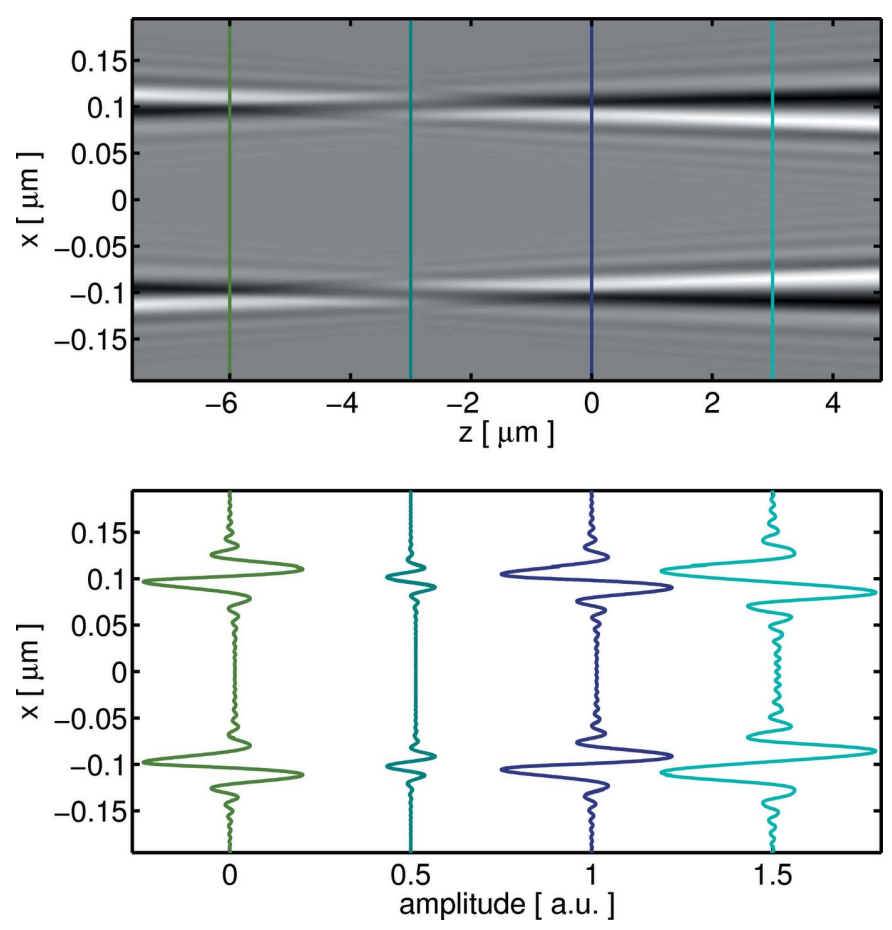

Figure 3

Free-space propagation of the simulated complex-valued field of an empty $z_{c}=6 \mu \mathrm{m}$ high channel. The complex field at the exit of the channel $(z=0 \mu \mathrm{m})$ is propagated through free space in the forward (X-ray beam) and backward directions, and its amplitude is displayed as a gray-scale image (top) and in four sections (bottom) - the latter are offset in amplitude for clarity. The amplitude at the channel exit already exhibits Fresnel fringes, which become further pronounced upon forward propagation. In the Fraunhofer far-field regime, they will have developed into the diffraction orders. Free-space back propagation by half the channel height, i.e. to $z=-3 \mu \mathrm{m}$, considerably reduces the Fresnel fringes. 
is symmetric in $q$ due to the symmetry of the cosine but it is generally not real-valued unless $E(x)$ itself is real. Therefore, the only a priori information about the phases in reciprocal space is their symmetry with respect to $q=0$. An intrinsic ambiguity is the sign of the phase of the wavefield since both $E$ and the complex conjugate $E^{*}$ yield the same absolute value of the Fourier transform and thereby the same diffraction pattern.

4.1.5. Constraints. The constraints are needed to define the focal plane and thereby to converge to an unique solution, see \$4.1.2. Additionally, in case of a complex-valued object only $N$ measured amplitudes are known, opposed to $2 N$ parameters, namely amplitude and phase. This means that the problem is without constraints or further data underdetermined.

Often it is assumed that the reconstructed object is either purely absorbing or phase shifting and that positivity constraints can be applied. Strictly speaking, this is never true since any realistic object will alter both amplitude and phase of the incident X-ray wave. In our case of diffraction by a grating-like structure in transmission geometry, positivity cannot be used even in an approximate way since a truly complex valued object has to be reconstructed, $c f$. also the discussion of Fig. 8 in $\S 5$.

Another constraint commonly used is the support constraint, assuming that the object is located within a typically convex support area and that there is nothing outside this region, i.e. all amplitudes outside the support can be set to zero. Fluid confined in a microfluidic channel may be seen as the object and the region of the confining silicon walls as the area outside the support. In this area, the electron density is constant but definitely not zero, i.e. we need a modified support constraint.

Even though a complex object has to be reconstructed, the number of parameters can nevertheless be almost halved by coupling amplitude and phase in a large part of the reconstructed profile. This is possible since we know the real and imaginary parts of the refractive index [equation (1)] for both the confining silicon walls and the confined disordered colloidal fluid. The phase shift $\Delta \Phi$ caused by traversing the length $\Delta z$ of a medium with real part of the refractive index $\delta$ is given by

$$
\Delta \Phi(x)=k \delta(x) \Delta z
$$

The attenuation coefficient $\mu$ in the same material is calculated from the imaginary part of the refractive index $\beta$ as $\mu=2 k \beta$. The attenuation of the wave follows the exponential law $I(z+\Delta z) / I(z)=\exp (-\Delta z \mu)$. Using equation (2), one can calculate the change in amplitude from the phase shift:

$$
\frac{|E(x, z+\Delta z)|}{|E(x, z)|}=\exp \left[-\Delta \Phi(x) \frac{\beta(x)}{\delta(x)}\right],
$$

assuming that the first Born approximation can be applied. In each iteration of the phase retrieval, the amplitude can be calculated from the phase in this way. The phase retrieval will work without this coupling at least for smaller ratios of the gap size to the period of the array, i.e. for smaller numbers of free parameters. See $\$ 4.1 .6$ for a discussion.
In equation (7), $\delta(x)$ and $\beta(x)$ are assumed to be constant, i.e. we are working (locally, for each discrete value of $x$ ) with the homogeneous object approach. Therefore, minor changes of $\delta(x)$ and $\beta(x)$,e.g. due to ordering of the colloidal particles, are seen as apparent changes of the sample thickness $\Delta z$. Vice versa, one can calculate the refractive index from the phase under the assumption that the height of the grating structures is constant, $c f$. $\S 4.1 .8$ and equation (8).

Owing to the ambiguity of the sign of the phase shift $\Delta \Phi$, it may be necessary to use $-\Delta \Phi$ rather than $\Delta \Phi$ to calculate the amplitude using equation (7).

The iterative phase retrieval described here was performed on a relative scale using the region of the confining silicon walls as baseline. Amplitude and phase are given relative to the change induced by the walls and the refractive-index values used for equation (7) are implemented as the deviation from the values for silicon.

The following constraints are used for our MAPP technique.

(i) The reconstructed complex field $E(x)$ has to be symmetric with respect to $x=0$.

(ii) The region of the object $\mathcal{O}$ and a non-object region $\mathcal{N}$ are pre-defined. A few pixels in the area of the confining walls $\mathcal{W}$ do not belong to any of these two sets and remain unconstrained. See Fig. 4 for a sketch.

(iii) The phase of the exit field is zero in the non-object region $\mathcal{N}$ and the amplitude constant.

(iv) In the object region $\mathcal{O}$, amplitude and phase are coupled via equation (7).

(v) The phase variations in the object region $\mathcal{O}$ are limited to, for example, $\pm 0.1 \mathrm{rad}$ relative to the average value.

(vi) If the average phase in the object region $\mathcal{O}$ does not have the pre-chosen sign, then the sign of the phase is inverted.

The symmetry constraint (i) is well justified since the ensemble average is retrieved and on average the rather perfect gratings and the fluid inside will exhibit a symmetric profile. Finding the regions for constraint (ii) is straightforward since the algorithm will only find a solution if this is done correctly, i.e. a fast trial-and-error procedure is successful. To be precise, the definition of these regions by itself is not a constraint, instead, the following constraints are applied to these regions. The assumption that the amplitude is equal throughout the non-object region, constraint (iii), is justified

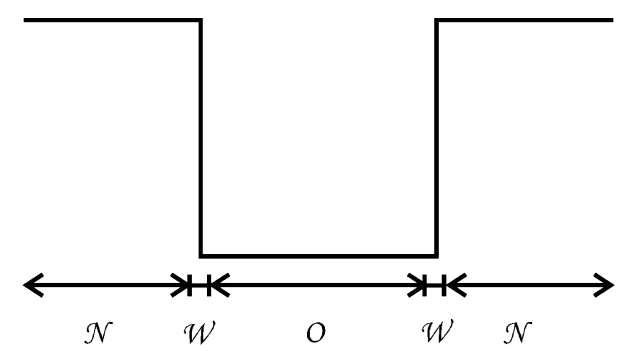

Figure 4

Sketch of the three regions for constraint (ii): object $\mathcal{O}$, non-object $\mathcal{N}$ and the wall $\mathcal{W}$ in between. 
since the silicon outside the channels is unstructured and has uniform thickness. Setting the phase in this region to zero defines just the baseline since all phase changes are measured relative to the change in this region. Using the assumption of an unstructured wall region in an iterative algorithm is similar to direct-space solvent flattening used in crystallography, i.e. the assumption of unstructured solvent regions within the unit cell (Wang, 1985). The coupling of amplitude and phase, constraint (iv), can be performed using the average $\delta$ and $\beta$ values for the solution since only minor density fluctuations are to be detected. In the present implementation, the amplitude is calculated from the phase in every iteration. The range constraint, number (v), helps initially to retrieve the basic shape and thereby the depth of the channels within a few iterations. Care has been taken that the allowed phase range is well above the magnitude of the phase oscillations which are finally retrieved in the object region. The selection of a sign, constraint (vi), is again not really a constraint since the sign of the reconstructed phase is ambiguous, as mentioned above. Preferring one sign over the other just ensures that the coupling of amplitude and phase can rely on a certain sign convention and it eases averaging of solutions as described in $\S 4.1 .7$.

We would like to point out that the precise position of the confining walls and thereby the width of the channels, the height of the channels and the phase profile of the fluid inside the channels are not constrained. This approach is therefore truly model-independent: as much prior information as is available is used but none of the parameters to be determined is imposed in any way onto the system.

4.1.6. Oversampling. Bragg sampling by measuring diffraction peaks is usually treated separately from the oversampling by recording intensity information at higher frequencies, i.e. also between the diffraction peaks or in general by sampling a speckle pattern of an arbitrary nontranslation periodic object. Nevertheless, there can be strong similarities between these cases as discussed in the following.

We define the degree of oversampling $D$ as the number of independent data points divided by the number of parameters to be determined. Mirror symmetry halves in the present case both the number of independent data points to $N / 2$ and the number of parameters to be determined. We therefore neglect this factor two in the following.

With the nomenclature used in Fig. 4, the number of points within the translation-invariant subunit $\mathcal{P}$ is given by $\mathcal{P}=\mathcal{N}+\mathcal{W}+\mathcal{O}$. With no free parameters in the non-object region $\mathcal{N}$, amplitude and phase as free parameters in the wall region $\mathcal{W}$ and coupled amplitude and phase in the object region $\mathcal{O}$, one gets for the degree of oversampling:

$$
D=\frac{\mathcal{N}+\mathcal{W}+\mathcal{O}}{O+2 W}=\frac{\mathcal{P}}{\mathcal{P}-\mathcal{N}+\mathcal{W}}>1 \text { for } \mathcal{N}>\mathcal{W}
$$

The non-object region is always larger than the wall region, i.e. the degree of oversampling is always greater than one.

If the amplitude and phase are not coupled in the object region then

$$
D=\frac{\mathcal{N}+\mathcal{W}+\mathcal{O}}{2 \mathcal{W}+2 \mathcal{O}}=\frac{P}{2(P-N)}>1 \text { for } \quad \mathcal{N}>\mathcal{P} / 2
$$

This is the standard oversampling criterion, that half the object must be known. In the present case, this means that the gap size of the channels must be smaller than half the period of the channel array if amplitude and phase are not coupled within the object region $\mathcal{O}$.

From these considerations, it follows that in the present case more diffraction data are available than non-constant pixels in the reconstructed field, i.e. the problem is overdetermined and in this sense the data are oversampled - even though Bragg sampling is used. The same is valid if the unit cell of a crystal contains parts of constant electron density like it is used in the solvent-flattening method (Wang, 1985). Furthermore, the individual values of the field or electron density are usually not completely free parameters. 'Soft constraints' can be used, i.e. knowledge about the system limits the parameter space. An example is the allowed phase range within the object region, constraint (v).

We conclude that the criteria for oversampling or for a problem being overdetermined may be less well defined than one may expect at first sight and that there is a smooth transition between Bragg sampling of diffraction data and oversampling of continuous data. This is also reflected by the strong similarity of some data analysis algorithms used in crystallography and for example lensless imaging, $c f$. 4.1.3.

The uniqueness of a solution can for example be shown by statistical means like averaging many similar solutions, see $\S 4.1 .7$.

4.1.7. Statistics. It is important to ensure that a unique solution has been found. Additionally, one would like to determine the error bar on the retrieved profiles. To achieve both objectives, several solutions are averaged.

The iterative phase retrieval was started for each data set, i.e. for each microfluidic array, 150 times with an initially random solution. For each of the refined 150 solutions, the similarity to all other solutions was calculated in the form of the mean-squared deviation (MSD), see Fig. 5. The refined solutions are grouped into a large set of similar solutions and a small set of dissimilar ones. The level of rejection is the median value of the MSDs plus their standard deviation, as indicated by the horizontal line in Fig. 5. It turns out that the dissimilar solutions differ from the majority of the solutions in the intermediate wall region that is unrestricted in the iterative phase-retrieval algorithm, $c f$. $\$ 4.1 .5$. Of the majority of similar solutions, the average result is calculated. Typically less than $10 \%$ of the initial 150 solutions are rejected, i.e. for all the data presented here, well above 100 solutions are averaged.

4.1.8. Concentration profiles. The phase profile of the $\mathrm{X}$-ray beam across the microfluidic channels can be determined in a model-independent way, as described in the previous sections. To convert the phase profiles into concentration profiles, we use our knowledge about the refractive indices of the components of the system under investigation. In the example presented here, the components are: silicon, $\delta_{\mathrm{Si}}=3.497 \times 10^{-6}, \beta_{\mathrm{Si}}=3.832 \times 10^{-8}$; silica colloidal 
particles, $\delta_{\text {silica }}=2.889 \times 10^{-6}, \beta_{\text {silica }}=1.776 \times 10^{-8}$; and a refractive-index-matched solvent of $55 \%$ benzyl alcohol and $45 \%$ ethanol, $\quad \delta_{\text {solvent }}=1.521 \times 10^{-6}$ and $\beta_{\text {solvent }}=$ $1.301 \times 10^{-9}$. The $\delta$ and $\beta$ values are given for the $\mathrm{X}$-ray wavelength $\lambda=1.050 \AA$. The $\delta$ values of the colloidal solution relative to the confining silicon can be calculated from the volumetric content $c$ of the colloidal particles: $\delta(x)=$ $c(x) \delta_{\text {silica }}+[1-c(x)] \delta_{\text {solvent }}-\delta_{\mathrm{Si}}$. Additionally, we know the height of the microfluidic channels of $z_{c}=6 \mu \mathrm{m}$ by design and also from the measurements of the empty channels. Therefore, we can use equation (6) to convert the determined phase shift inside the gap $\Delta \Phi_{\text {rel }}$, relative to the surrounding silicon, into a concentration of colloidal particles $c_{\text {silica }}$ :

$$
c_{\text {silica }}=\frac{\delta_{\mathrm{Si}}+\frac{\Delta \Phi_{\mathrm{rel}}}{k z_{c}}-\delta_{\text {solvent }}}{\delta_{\text {silica }}-\delta_{\text {solvent }}} .
$$

Equation (8) can only be applied to binary mixtures. The concentration profiles shown in $\$ 5$ are based on this conversion. Using the amplitude will not yield additional information since the amplitude has been calculated from the phase, see $\S 4.1 .5$.

\section{Results}

To allow the reader to estimate the precision and uniqueness of the methods, some experimental results are reported here. The system chosen is a colloidal solution of 10 volume percent
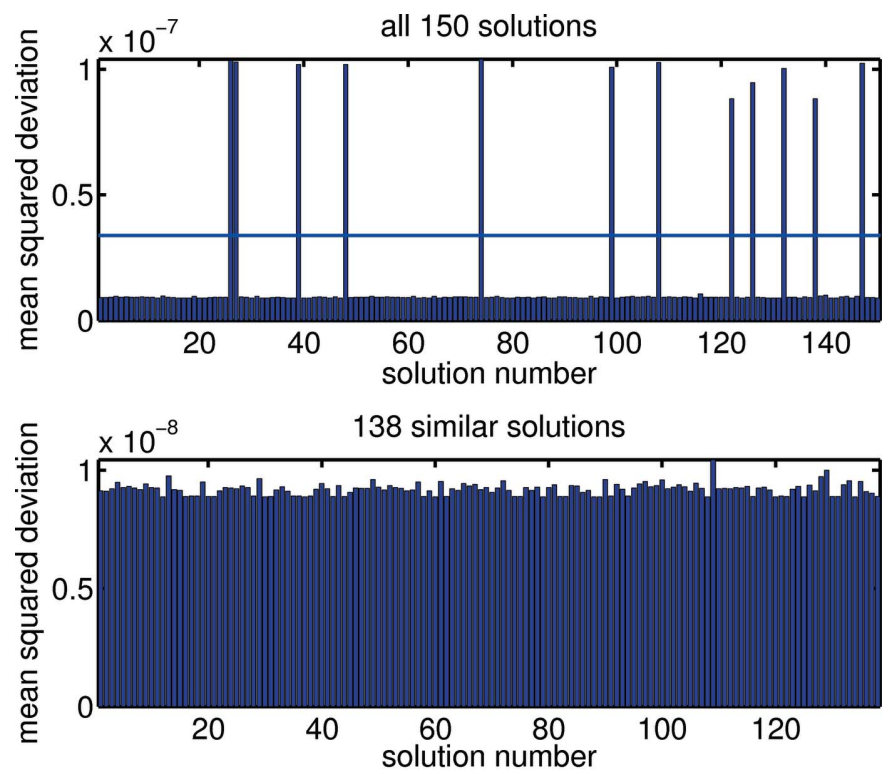

Figure 5

Selecting similar solutions. The iterative phase retrieval was started 150 times with a random initial state. For each of the refined 150 solutions, the similarity to all other solutions was calculated in the form of the mean squared deviation (MSD). The MSD is shown as a bar chart in the top panel. All solutions with a MSD higher than one standard deviation above the median of the MSD were rejected. This limit is marked with a horizontal line in the top panel. This procedure leads to the much more uniform MSD distribution shown in the bottom panel. From these (in the present case) 138 solutions, the average solution was calculated. silica spheres of $109.2 \mathrm{~nm}$ average diameter in a refractiveindex-matched solvent of $55 \%$ benzyl alcohol and $45 \%$ ethanol for a first measurement, and with additionally lithium chloride $(\mathrm{LiCl})$ in $0.4 \mathrm{M}$ concentration for a second measurement. The matching of the refractive index for the solution reduces differences in the electric susceptibility and thereby the van der Waals interactions between the colloidal particles. The additional $\mathrm{LiCl}$ ions in the salt solution shield the electrostatic potential of the charged colloid particles. The silica colloid particles then effectively become a hard-sphere system: the paradigm of a model fluid. The polydispersity of the silica spheres is approximately $3 \%$. The concentration is well below the density for the formation of a crystalline layer at the wall or even capillary freezing (Dijkstra, 2004).

\subsection{Phase profiles, uniqueness, resolution}

The determined phase profiles for the first measurements with charged colloids and the second measurements after the addition of salt but still with the same microfluidic gratings are depicted in Fig. 6. The channel widths shown for each profile are determined as the full width at half average maximum of
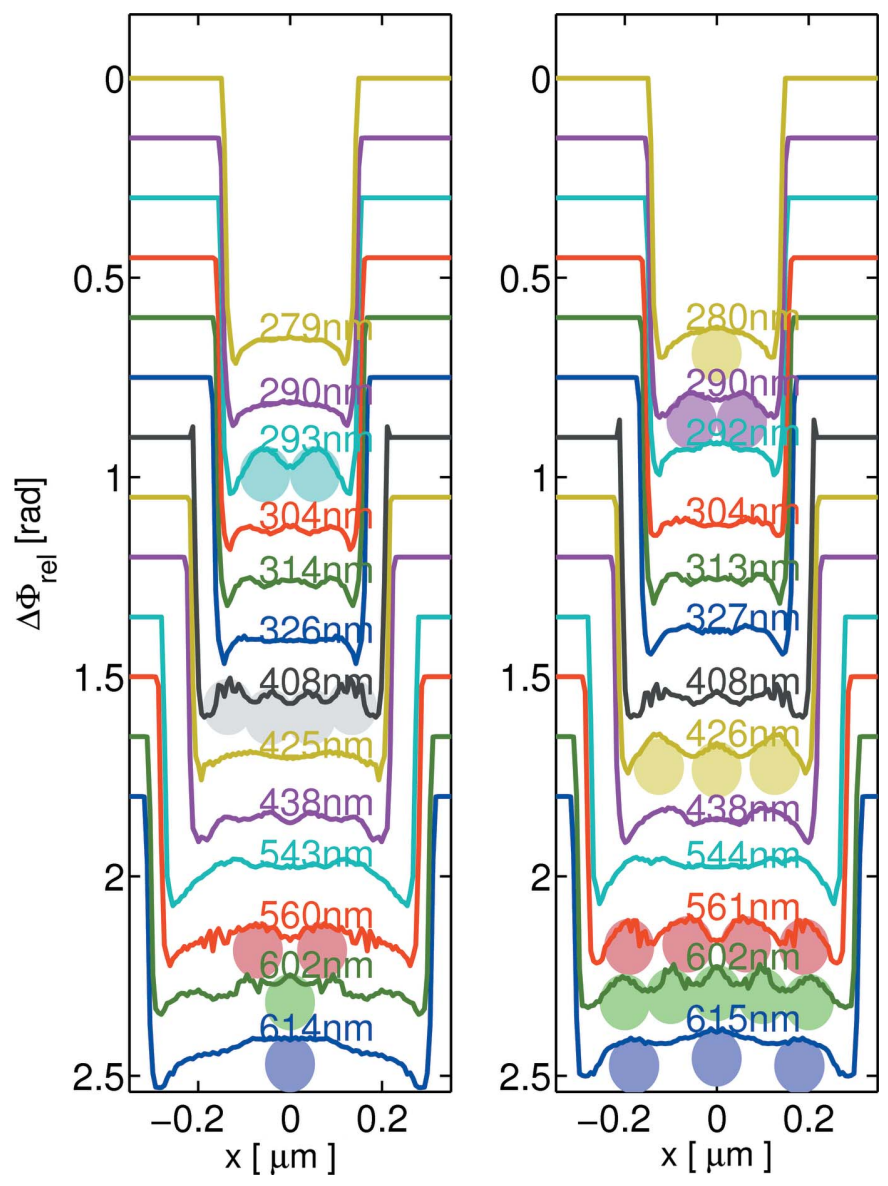

Figure 6

Phase profiles of charged silica colloids (left) and hard-sphere-like colloids (right) in the same microfluidic arrays. The profiles are offset vertically for clarity. The determined channel widths are shown for each profile and layering, i.e. pronounced increased concentration, is indicated by spheres. 
the phase profile. These values agree between the two data sets for the same array to within $1 \mathrm{~nm}$, which is much better than the minimal single-pixel resolution of $6 \mathrm{~nm}$ and demonstrates the precision of the method and the uniqueness of the phase-retrieval results.

The only remaining uncertainty is the distinction between a solution and its Babinet solution. This means for channels with a period $p$ and a width $w$ it is difficult to distinguish between the solution with the correct width $w$ and the Babinet solution of width $p-w$. This ambiguity could at least partially be removed if the intensities were recorded on an absolute scale.

An example of the excellent agreement between measured and retrieved intensities is shown in Fig. 7. The data are reconvoluted with the Gaussian peak, $c f$. $\S 3$, to resemble the original data. Intensities up to a momentum transfer $q$ of $0.5 \mathrm{~nm}^{-1}$ were used, which corresponds to up to $(N-1) / 2+1=100$ diffraction orders in the case of the gratings with $1.25 \mu \mathrm{m}$ period. The symmetry of the system adds a factor of two and the resulting pixel size is approximately $6 \mathrm{~nm}$ in direct space. The inset shows the deviations that occur for the highest orders due to the averaging of solutions. There are considerable deviations for a few diffraction orders but most of the intensities are reproduced well, even at high momentum transfer. Therefore, we conclude

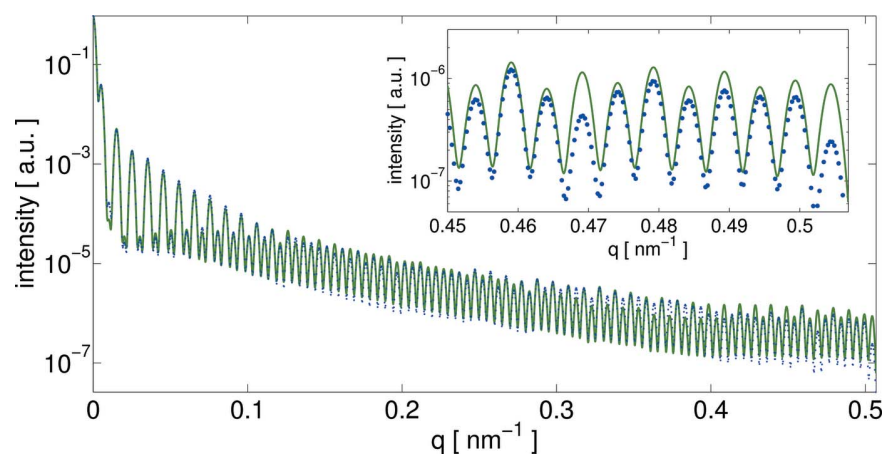

Figure 7

Experimental data. Retrieved (green line) and measured (blue dots) intensities on a logarithmic scale for 100 diffraction orders of a grating with $1.25 \mu \mathrm{m}$ period and $615 \mathrm{~nm}$ channel width.
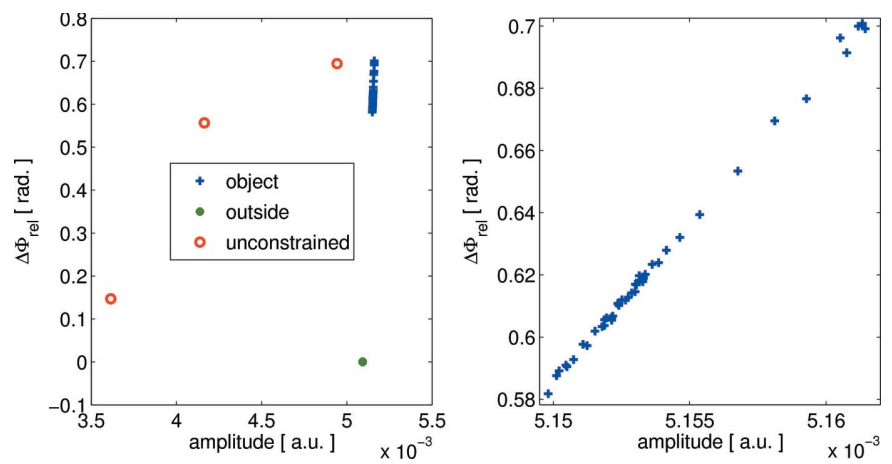

Figure 8

Retrieved amplitude and phase plotted in the plane of complex numbers for the complete profile (left) and the section specific to the object, i.e. the colloidal solution (right). that twice the pixel size of $6 \mathrm{~nm}$ in the present case is also the resolution.

That the average of many (for the displayed example 138) solutions reproduces the measured data so well proves that the one-dimensional phase retrieval leads to unique solutions.

It is instructive to plot amplitude and phase for a determined profile in the complex plane, see Fig. 8. The points along the profile inside the silicon walls all have the same amplitude/phase relation and fall onto a single point marked 'outside' in the legend. Six points are in the region directly at the wall of the channel. These points do not follow a simple amplitude/phase relationship and can be seen as a local deviation from the simple Born approximation. All points for the colloidal solution inside the gap fall onto a single line, as shown more clearly in the right panel of Fig. 8. The colloidal solution can be seen as a binary mixture of fluid and colloidal particles and all amplitude/phase points fall onto the line connecting the point corresponding to the minimum concentration of colloidal particles towards the point corresponding to the maximum concentration.

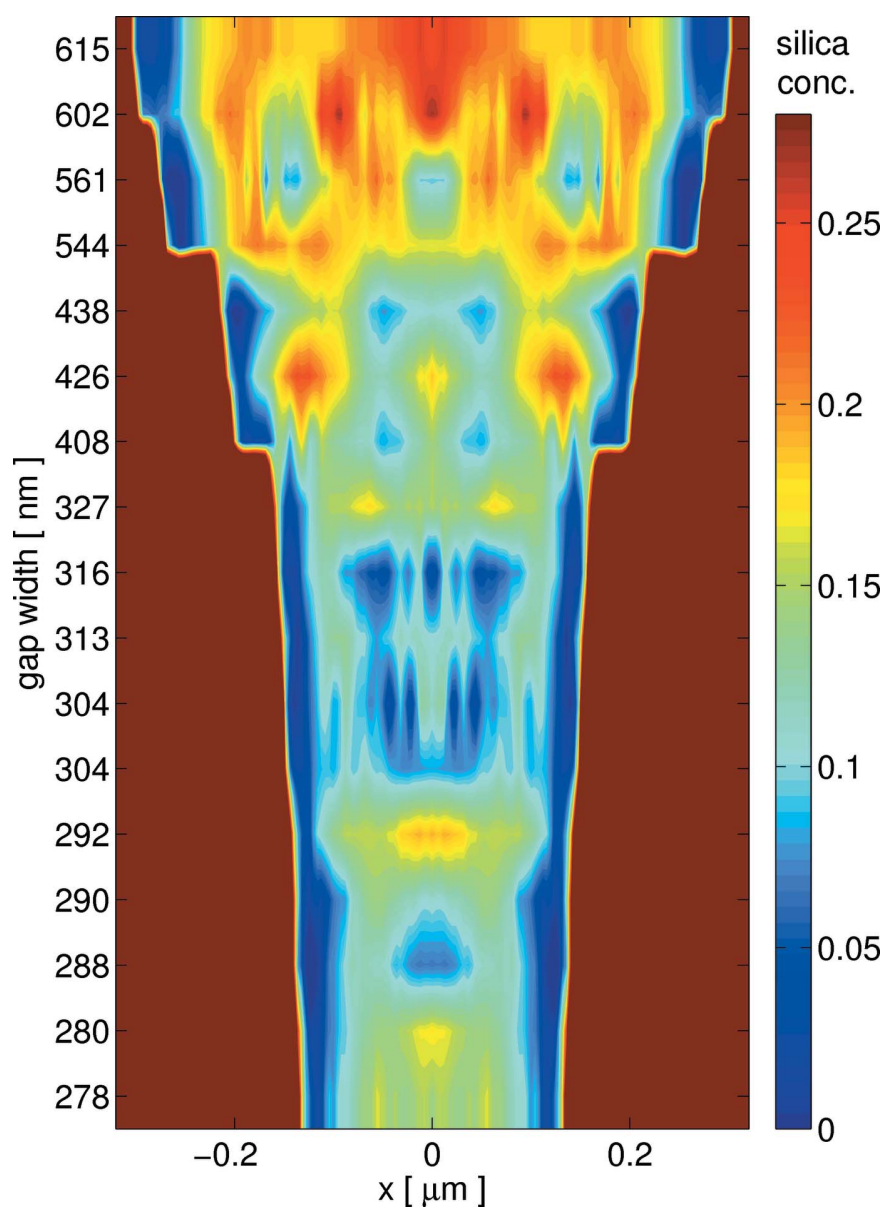

Figure 9

The silica concentration inside the nanofluidic channels is shown as a contour plot for several channel widths. The excluded volume effect of $0 \%$ concentration at the confining walls as well as pronounced concentration increase due to the confinement are clearly visible. The dark red outer region is the area of the confining silicon walls. 


\subsection{Concentration profiles}

Phase profiles can directly be converted to concentration profiles for the colloidal particles, as described in \$4.1.8. As an example, the phase profiles for the hard-sphere system, shown in the right part of Fig. 6, are displayed as a concentration plot in Fig. 9.

The concentration of the silica spheres, spatially resolved across the confining channels, varies from $0 \%$ due to the excluded volume effect close to the walls to more than $25 \%$ in regions of high concentration. In addition, it is found that the average density across the channel width oscillates in phase with the degree of order. This is not unexpected, since the layering effect allows for a denser packing of the particles. The channels are in contact with a reservoir between the glass plate and the chip and these changes of the average density can therefore occur.

For the hard-sphere fluid, pronounced layering in two, three and four layers occurs at multiples of approximately $142 \mathrm{~nm}$ in channel width, i.e. at 290,426 and $561 \mathrm{~nm}$ (indicated by spheres in Fig. 6). This is a clear fingerprint of confinementinduced oscillatory ordering. Pronounced ordering is observed only if the given channel width fits to the layering distance of the colloid inside. The data unequivocally show that the oscillatory structural forces are directly linked to a sequence of layering-disordering transitions.

A more detailed discussion of the results on the colloidal systems is beyond the scope of this work and is published elsewhere (Bunk et al., 2007).

\section{Summary and outlook}

Using microfluidic arrays as sample containers for studies of fluids under confinement allows for a low radiation dose and the ensemble average is studied rather than individual entities. A novel one-dimensional X-ray phase-retrieval approach applied to X-ray diffraction data from such microfluidic arrays provides unique solutions determined in a model-independent way. As an example, concentration profiles for colloidal particles with a resolution in the $10 \mathrm{~nm}$ range have been presented.

Scaling down the channels in these arrays to the range of $10-50 \mathrm{~nm}$ will offer unique opportunities for studies of confinement-induced ordering of proteins in solution.

This work was performed at the Swiss Light Source, Paul Scherrer Institut, Villigen, Switzerland. Celestino Padeste, Heilke Keymeulen, Philip R. Willmott, Bruce D. Patterson, Hua Guo and Gerard H. Wegdam contributed in different ways to the measurements on colloidal solutions (Bunk et al., 2007). We thank Dominik Meister and Michael Lange for technical support and Harun H. Solak and C. Theiler for fruitful discussions.

\section{References}

Bruck, Y. M. \& Sodin, L. G. (1979). Opt. Commun. 30, 304-308.

Bunk, O., Diaz, A., Pfeiffer, F., David, C., Padeste, C., Keymeulen, H., Willmott, P., Patterson, B., Schmitt, B., Satapathy, D., van der Veen, J. F., Guo, H. \& Wegdam, G. (2007). Phys. Rev. E, 75, 021501.

Chapman, H. N., Barty, A., Marchesini, S., Noy, A., Hau-Riege, S. P., Cui, C., Howells, M. R., Rosen, R., He, H., Spence, J. C. H., Weierstall, U., Beetz, T., Jacobsen, C. \& Shapiro, D. (2006). J. Opt. Soc. Am. A, 23, 1179-1200.

Diaz, A. (2006). X-ray Scattering Studies of Ordering Phenomena in Colloids Confined in Microcavity Arrays. PhD thesis, No. 16851, ETH Zurich, Switzerland.

Diaz, A., David, C., Guo, H., Keymeulen, H., Pfeiffer, F., Wegdam, G., Weitkamp, T. \& van der Veen, J. F. (2005). Physica (Utrecht) B, 357, 199-203.

Diaz, A. \& van der Veen, J. F. (2007). Thin Solid Films, 515, 5645-5653.

Dijkstra, M. (2004). Phys. Rev. Lett. 93, 108303.

Elser, V. (2003). J. Opt. Soc. Am. A, 20, 40-55.

Fienup, J. R. (1982). Appl. Opt. 21, 2758-2769.

Gerchberg, R. W. (1986). Optik, 74, 91-93.

Gerchberg, R. W. \& Saxton, W. O. (1972). Optik, 35, 237-246.

Israelachvili, J. N. (1991). Intermolecular and Surface Forces, 2nd ed. New York: Academic Press.

Israelachvili, J. N. (1992). Surf. Sci. Rep. 14, 109-159.

Miao, J., Charalambous, P., Kirz, J. \& Sayre, D. (1999). Nature (London), 400, 342-344.

Miao, J., Hodgson, K. O., Ishikawa, T., Larabell, C. A., LeGros, M. A. \& Nishino, Y. (2003). Proc. Natl Acad. Sci. USA, 100, 110-112.

Patterson, B. D., Abela, R., Auderset, H., Chen, Q., Fauth, F., Gozzo, F., Ingold, G., Kühne, H., Lange, M., Maden, D., Meister, D., Pattison, P., Schmidt, T., Schmitt, B., Schulze-Briese, C. et al. (2005). Nucl. Instrum. Methods Phys. Res. A, 540, 42-67.

Pfeifer, M. A., Williams, G. J., Vartanyants, I. A., Harder, R. \& Robinson, I. K. (2006). Nature (London), 442, 63-66.

Quiney, H. M., Peele, A. G., Cai, Z., Paterson, D. \& Nugent, K. A. (2006). Nature Phys. 2, 101-104.

Schmitt, B., Brönnimann, C., Eikenberry, E. F., Hülsen, G., Toyokawa, H., Horisberger, R., Gozzo, F., Patterson, B., Schulze-Briese, C. \& Tomizaki, T. (2004). Nucl. Instrum. Methods Phys. Res. A, 518, 436-439.

Seeck, O. H., Kim, H., Lee, D. R., Shu, D., Kaendler, I. D., Basu, J. K. \& Sinha, S. K. (2002). Europhys. Lett. 60, 376-382.

Shapiro, D., Thibault, P., Beetz, T., Elser, V., Howells, M., Jacobsen, C., Kirz, J., Lima, E., Miao, H., Neiman, A. M. \& Sayre, D. (2005). Proc. Natl Acad. Sci. USA, 102, 15343-15346.

Spence, J. C. H., Weierstall, U. \& Howells, M. (2002). Philos. Trans. $R$. Soc. London Ser. A, 360, 875-895.

Thibault, P., Elser, V., Jacobsen, C., Shapiro, D. \& Sayre, D. (2006). Acta Cryst. A62, 248-261.

Vartanyants, I., Ern, C., Donner, W. \& Dosch, H. (2000). Appl. Phys. Lett. 77, 3929-3931.

Walther, A. (1963). Opt. Acta, 10, 41-49.

Wang, B. (1985). Methods Enzymol. 115, 90-112.

Zwanenburg, M. J., Peters, J. F., Bongaerts, J. H. H., de Vries, S. A., Abernathy, D. L. \& van der Veen, J. F. (1999). Phys. Rev. Lett. 82, 1696-1699. 